\title{
Erratum to: RNA interference-mediated knockdown of RhoGDI2 induces the migration and invasion of human lung cancer A549 cells via activating the PI3K/Akt pathway
}

\author{
Huiyan Niu • Baogang Wu • Yang Peng • \\ Hongfang Jiang • Yi Zhang • Jiahe Wang • Yifei Zhang • \\ Ping He
}

Published online: 10 December 2014

(C) International Society of Oncology and BioMarkers (ISOBM) 2014

\section{Erratum to: Tumor Biol}

DOI 10.1007/s13277-014-2671-9

The authors missed in including the Acknowledgement section for this paper. The statement is provided below.

Acknowledgments This work was supported by grants from the National Natural Science Foundation of China (No.: 81201832), the Outstanding Scientific Fund of Shengjing Hospital, and the Specialized Research Fund for the Doctoral Program of Higher Education (No.: 20122104110011).

The online version of the original article can be found at http://dx.doi.org/ 10.1007/s13277-014-2671-9.

H. Niu $\cdot$ B. Wu $\cdot$ Y. Peng $\cdot$ H. Jiang $\cdot$ Y. Zhang $\cdot$ J. Wang $\cdot$

Y. Zhang $\cdot$ P. He $(\bowtie)$

Department of Geriatrics, Shengjing Hospital of China Medical University, 36 Sanhao Street, Shenyang 110004, People's Republic of China

e-mail: hep@sj-hospital.org 